\title{
Influência do traumatismo dentário na qualidade de vida de crianças de 8 a 10 anos do município de Patos - PB, Brasil
}

Influence of dental trauma on the quality of life of children from 8 to 10 years old of the city of Patos-PB, Brazil Influencia del trauma dental en la calidad de la vida de los hijos de 8 a 10 años en la municipio de Patos-PB, Brasil Jéssica Fernanda Delfino dos SANTOS ${ }^{1}$

Larissa Rafaela de Medeiros SILVA ${ }^{1}$

Elaine Roberta Leite de SOUZA ${ }^{1}$ Natália Magalhães ALVES ${ }^{1}$

Tássia Cristina de Almeida Pinto SARMENTO²

${ }^{1}$ Curso de Graduação em Odontologia. Centro de Saúde e Tecnologia Rural, Universidade Federal de Campina Grande - UFCG 58708-110 Patos/PB, Brasil

${ }^{2}$ Professora Doutora do Curso de Graduação em Odontologia. Centro de Saúde e Tecnologia Rural, Universidade Federal de Campina Grande - UFCG 58708-110 Patos/PB, Brasil

\section{Resumo}

Introdução: O traumatismo dentário é consequência de injúrias ao órgão dental que ocorrem ocasionalmente. A etiologia principal são quedas e acometem principalmente os incisivos centrais superiores, ocasionando fratura em esmalte e dentina, tendo como maiores vítimas indivíduos na primeira década de vida. Os traumas dentários são capazes de causar grandes impactos psicológicos e sociais, que influenciam diretamente na qualidade de vida do indivíduo. Objetivo: Avaliar a influência do traumatismo dentário na qualidade de vida de crianças de 8 a 10 anos de idade, que frequentam escolas públicas situadas no município de Patos-PB, Brasil. Metodologia: Trata-se de um estudo observacional, quantitativo, analítico e de corte transversal, com amostra aleatória e representativa, totalizando 384 crianças. Os dados foram coletados mediante aplicação do questionário $\mathrm{CPQ}_{8-10}$, sendo após realizado exame clínico por pesquisadores calibrados (Kappa intraexaminadores, 0,91; Kappa interexaminadores, 0,84). Os resultados foram tabulados em um banco de dados e calculados através do programa SPSS (statical package for social sciences) versão 8.0. Resultados: A ocorrência de trauma foi predominante no sexo masculino, tendo acometido mais o grupo dos dentes incisivos. O tipo de trauma mais frequente foi a fratura de esmalte e verificou-se associação estatística do trauma dental com a idade ( $\mathrm{p}=0,042)$. Houve impacto dos traumatismos dentários na qualidade de vida $(\mathrm{p}=0,011)$. Conclusão: Diante da elevada prevalência dos traumatismos dentários, observou-se impacto na qualidade de vida das crianças, necessitando assim de mais estudos que avaliem essa associação para que estratégias preventivas possam ser adotadas.

Descritores: Traumatismos Dentários; Qualidade de Vida; Criança.

\section{Abstract}

Introduction: Dental trauma is a consequence of dental organ damage that occurs occasionally. The main etiology is falls and mainly affect the upper central incisors, causing enamel and dentin fracture, having as major victims individuals in the first decade of life. Dental trauma can cause great psychological and social impacts, which directly influence in the individual's quality of life. Objective: This study aimed to evaluate the influence of dental trauma on the quality of life of children from 8 to 10 years old, who attend public schools in the city of Patos-PB, Brazil. Methodology: This is an observational, quantitative, analytical and cross-sectional study, with a random and representative sample, totaling 384 children. Data were collected by applying the CPQ8-10 questionnaire, and after clinical examination by calibrated researchers (intra-examiner Kappa, 0.91; interexaminer Kappa, 0.84). Results were tabulated in a database and calculated using the program SPSS (statical package for social sciences) version 8.0. Results: The occurrence of trauma was predominant in males, and most affected group of incisors, The most frequent type of trauma was enamel fracture and there was a statistical association between dental trauma and age ( $p$ $=0.042)$. There was dental trauma impact of quality of life $(p=0.011)$. Conclusion: Given the high prevalence of dental trauma, there was an impact on the quality of life of children, thus requiring further studies to evaluate this association so that preventive strategies can be adopted.

Descriptors: Tooth Injuries; Quality of Life; Child.

\section{Resumen}

Introducción: El trauma dental es una consecuencia de las lesiones en el órgano dental que ocurren ocasionalmente. La etiología principal es la caída y afecta principalmente a los incisivos centrales superiores, causando fractura en el esmalte y la dentina, teniendo como grandes víctimas individuos en la primera década de vida. Los traumas dentales son capaces de causar grandes impactos psicológicos y sociales, que influyen directamente en la calidad de vida del individuo. Objetivo: Evaluar la influencia del trauma dental en la calidad de vida de los niños de 8 a 10 años, que asisten a escuelas públicas ubicadas en el municipio de Patos-PB. Metodología: Se trata de un estudio observacional, cuantitativo, analítico y transversal, con una muestra aleatoria y representativa, que suma 384 niños. Los datos se recopilaron mediante la aplicación del cuestionario CPQ8-10, siendo después del examen clínico por investigadores calibrados (Intraexaminador Kappa, 0.91; Interexaminadores Kappa, 0.84). Los resultados se tabularon en una base de datos y se calcularon utilizando el programa SPSS (statical package for social sciences) versión 8.0. Resultados: La ocurrencia de trauma fue predominante en los varones, habiendo afectado más el grupo de dientes incisivos, el tipo más frecuente de trauma fue la fractura por esmalte y hubo asociación estadística de Trauma Dental con la edad ( $P$ - 0,042). Hubo un impacto de las lesiones dentales en la calidad de vida $(P-0,011)$. Conclusión: Dada la alta prevalencia del trauma dental, hubo un impacto en la calidad de vida de los niños, necesitando así más estudios que evalúen esta asociación para que se puedan adoptar estrategias preventivas.

Descriptores: Traumatismos de los Dientes; Calidad de Vida; Niño.

INTRODUÇÃO

O traumatismo dentário é definido como consequência de uma injúria térmica, química ou mecânica, que acomete o dente e seus tecidos de suporte ${ }^{1}$. As suas principais causas são quedas, colisões contra objetos ou pessoas, práticas esportivas, violência e acidentes automobilísticos ${ }^{2}$.

O tipo mais comum de lesão provocada por trauma dental é a fratura de esmalte ou esmalte e dentina, predominante na dentição permanente ${ }^{3}$. Lesões que acometem a dentição decídua tendem a ser mais sérias ${ }^{4}$. Os 
elementos mais acometidos são os incisivos superiores centrais seguidos pelos laterais, devido a sua localização no arco dental ${ }^{5}$. Indivíduos na primeira década de vida e do gênero masculino apresentam maior prevalência de acometimento. Estes dados podem ser explicados pelo fato de crianças serem em sua maioria, curiosas e inquietas e não possuírem maturação motora suficiente para sua autoproteção ${ }^{6}$.

Quando ocorre um trauma dentário é necessário que condutas adequadas sejam tomadas de imediato, pois aumentam as chances de obter um bom prognóstico em relação ao dente, visto que falhas durante 0 socorro podem causar a perda do elemento dentário $^{7}$. Logo, traumas que acometem dentes anteriores podem causar danos na função e afetar a autoestima, o comportamento e o sucesso pessoal, tornando-se um fator de impacto negativo na qualidade de vida da criança e seus familiares ${ }^{8}$.

Para avaliar a qualidade de vida relacionada à saúde bucal, foram desenvolvidos instrumentos específicos, entre eles, estão questionários que são preenchidos pelos pais ou pela própria criança. Para a avaliação da qualidade de vida relacionada à saúde bucal de crianças de 8 a 10 anos, pode-se utilizar o Child Perceptions Questionnaire $\left(\mathrm{CPQ}_{8-10}\right)$ este instrumento, inclui aspectos gerais e específicos sobre as doenças bucais, ajustando-se aos conceitos de saúde infantil e direcionando-se aos diferentes estágios de desenvolvimento das crianças ${ }^{8}$.

O município de Patos-PB, Brasil, não apresenta dados epidemiológicos sobre traumatismos dentários e a influência destes na qualidade de vida relacionada à saúde bucal. $A$ partir dessa informação e pela gravidade do problema ser reconhecida em outras populações, o presente estudo objetivou investigar a influência do traumatismo dentário na qualidade de vida das crianças de 8 a 10 anos de idade, matriculadas em escolas públicas, no município de Patos-PB, Brasil.

\section{MATERIAL E MÉTODO}

De acordo com a resolução 466/12 do Conselho Nacional de Saúde (CNS), a realização do presente estudo iniciou-se após a análise e aprovação pelo Comitê de Ética em Pesquisa, via o sistema online da Plataforma Brasil, sendo aprovado mediante parecer de número: 2.982.122. Este estudo é do tipo observacional, quantitativo, analítico e de corte transversal, com amostra aleatória e representativa, correspondente a 384 escolares pertencentes às escolas públicas localizadas no
Município de Patos-PB, Brasil. Para a realização do cálculo amostral utilizou-se a fórmula de estimativa de proporção ${ }^{9}$.

Os critérios de inclusão da amostra foram: crianças regularmente matriculadas nas escolas públicas da cidade de Patos-PB, Brasil, na faixa etária de 8 a 10 anos, no decorrer da pesquisa, cujos pais e/ou responsáveis concordaram com a participação no estudo. Bem como, foram excluídas do estudo todas as crianças com deficiências neuropsicomotoras e crianças que estavam em tratamento ortodôntico no momento ou anteriormente ao exame clínico.

Para a avaliação do traumatismo dentário foi realizado exame clínico intrabucal e os dados foram anotados em uma ficha clínica previamente elaborada pelos pesquisadores devidamente calibrados (Kappa intraexaminadores, $\quad 0,91 ; \quad$ Kappa interexaminadores, 0,84). Durante o exame clínico, foi solicitado que a criança falasse sobre como ocorreu o trauma e em caso de dúvidas dos examinadores, perguntas sobre o trauma foram feitas com o objetivo de facilitar e definir o diagnóstico das lesões ou mesmo diferenciá-las de questões fisiológicas da dentição mista. A observação da cavidade oral foi realizada de forma direta com iluminação artificial (lanterna de cabeça com luz de LED - Diodo Emissor de Luz). Os instrumentais utilizados foram odontoscópios, sondas exploradoras no 5 , e gazes estéreis para secagem e limpeza da região. Para tal, as crianças ficaram sentadas em cadeiras escolares em uma sala da própria escola. Para o exame clínico o examinador usou equipamentos de proteção individual, sendo as luvas trocadas a cada exame e o gorro e a máscara a cada turno de exame.

Para avaliação do impacto na qualidade de vida foi utilizado o questionário $C P Q_{8-10}$, aplicado na forma de entrevista e individual. $O$ entrevistador lia as questões e as alternativas e a criança respondia para que o entrevistador marcasse a opção escolhida pela criança. O $\mathrm{CPQ}_{8-10}$ possui 29 questões de múltipla escolha abordando os impactos que as disfunções orais podem causar na qualidade de vida das crianças de 8 a 10 anos de idade ${ }^{10}$. Este instrumento possui quatro domínios, sintomas orais (questões de 5 a 9), limitações funcionais (questões de 10 a 14), bem-estar emocional (questões de 15 a 19), e bem-estar social (questões de 20 a 29). As questões de 5 a 29 são medidas com scores de 0 a 4 . As questões 1 e 2 são referentes ao gênero e idade respectivamente e as questões 3 e 4 referem-se a percepção global da saúde bucal e bem-estar 
geral e são medidas com scores de 0 a 3 . Sendo assim, o impacto das alterações bucais sobre a qualidade de vida das crianças é avaliado mediante a soma final dos escores do questionário. Valores altos, expressam alto impacto, valores baixos, expressam médio e baixo impacto ${ }^{11}$.

Para análise dos resultados do $C P Q_{8-10}$ foi realizado uma metodologia de categorização dos resultados em baixo impacto e alto/médio impacto de acordo com Cabral et al. ${ }^{12} \mathrm{e}$ Fernandes et al. $^{13}$, então considerando a soma de scores limite para o baixo impacto na qualidade de vida foi utilizado o terceiro quartil como ponto de corte, então para os domínios sintomas orais, limitações funcionais e bemestar emocional foi utilizado o ponto de corte 5, para o domínio bem-estar social, foi utilizado o ponto de corte 10 e para todos os domínios, foi utilizado o ponto 25. Acima desses valores, foi considerado alto e médio impacto na qualidade de vida das crianças.

As informações obtidas foram tabuladas em um banco de dados, e calculadas estatisticamente através do programa estatístico SPSS (Statistical Package for Social Sciences) na versão 8.0.

\section{RESULTADOS}

A amostra estudada de acordo com o cálculo amostral foi de 384 crianças, examinadas e entrevistadas, na faixa etária de 8 a 10 anos de idade. Observou-se predominância do sexo feminino $(54,7 \%, \mathrm{n}=210)$ e da idade de 9 anos $(37,8 \%, n=145)$.

No tocante a ocorrência do traumatismo dentário, verificou-se uma prevalência bastante elevada de $44 \%(n=169)$. Bem como a presença do impacto na qualidade de vida destas crianças também foi bastante significativa $(96,9 \%, \mathrm{n}=372)$.

Os traumas foram identificados em $97 \%$ dos casos nos dentes anteriores sendo estes incluindo os 4 incisivos superiores e inferiores. Destes traumas, o tipo mais prevalente foram as fraturas de esmalte $(65,8 \%, n=179)$, seguidas das luxações laterais $(11 \%, n=30)$ e menor porcentagem se verificou a luxação extrusiva $(0,36 \%, n=1)$.

Ao se analisar a associação entre o traumatismo dentário e o sexo, idade e impacto na qualidade de vida (Tabela 1), observa-se que os indivíduos do sexo masculino foram mais acometidos $(47,7 \%)$. É possível observar associação estatisticamente significante entre a presença do traumatismo dentário e a idade de 9 anos $(p=0,042)$, e a presença do impacto na qualidade de vida $(p=0,011)$. Ainda na
Tabela 1, observa-se que os traumatismos dentários foram mais prevalentes nos dentes incisivos, revelando associação estatisticamente significativa entre essas duas variáveis $(\mathrm{p}=0,000)$.

Tabela 1. Associação entre o traumatismo dentário e o sexo, idade e impacto na qualidade de vida

\begin{tabular}{|c|c|c|c|c|}
\hline \multicolumn{5}{|c|}{ TRAUMATISMO DENTÁRIO } \\
\hline Variável & Presente & Ausente & Total & Valor de p \\
\hline & $\mathrm{n}(\%)$ & $\mathrm{n}(\%)$ & $\mathrm{n}(\%)$ & \\
\hline \multicolumn{5}{|l|}{ Sexo } \\
\hline Feminino & $86(41,0)$ & $124(59,0)$ & $210(100,0)$ & \multirow{2}{*}{$0,185^{(1)}$} \\
\hline Masculino & $83(47,7)$ & $91(52,3)$ & $174(100,0)$ & \\
\hline \multicolumn{5}{|l|}{ Idade } \\
\hline 8 anos & $43(33,9)$ & $84(66,1)$ & $127(100,0)$ & \multirow{3}{*}{$0,042^{(2) *}$} \\
\hline 9 anos & $74(51,0)$ & $71(49,0)$ & $145(100,0)$ & \\
\hline 10 anos & $52(46,4)$ & $60(53,6)$ & $112(100,0)$ & \\
\hline \multicolumn{5}{|c|}{ Impacto na qualidade de vida } \\
\hline Presente & $168(45,2)$ & $204(54,8)$ & $372(100,0)$ & \multirow[b]{2}{*}{$0,011^{(1) *}$} \\
\hline Ausente & $1(8,3)$ & $11(91,7)$ & $12(100,0)$ & \\
\hline \multicolumn{5}{|c|}{ Grupo de dentes mais acometidos } \\
\hline Sem trauma & $0(0,0)$ & $215(100,0)$ & $215(56,3)$ & \multirow{4}{*}{$0,000^{*}$} \\
\hline Incisivos & $163(95,6)$ & $0(0,0)$ & $163(42,2)$ & \\
\hline Caninos & $3(1,8)$ & $0(0,0)$ & $3(0,8)$ & \\
\hline Pré-molares & $3(1,8)$ & $0(0,0)$ & $3(0,8)$ & \\
\hline
\end{tabular}

A Tabela 2 refere-se à associação entre os traumatismos dentários e impacto na qualidade de vida expressados pelos valores dos domínios do questionário $C P Q_{8-10}$. Observase que os domínios isoladamente não tiveram significância estatística com a presença dos traumatismos.

Tabela 2. Associação entre o traumatismo dentário e os domínios do questionário $C P Q_{8-10}$

\begin{tabular}{|c|c|c|c|c|}
\hline \multicolumn{5}{|c|}{ TRAUMATISMO DENTÁRIO } \\
\hline Variável & Presente & Ausente & Total & Valor de $\mathbf{p}^{(1)}$ \\
\hline & n (\%) & $\mathrm{n}(\%)$ & $\mathrm{n}(\%)$ & \\
\hline \multicolumn{5}{|l|}{ Domínio 1} \\
\hline Baixo impacto & $90(41,3)$ & $128(58,7)$ & $218(100,0)$ & \multirow[b]{2}{*}{0,217} \\
\hline Alto/médio impacto & $79(47,6)$ & $87(52,4)$ & $166(100,0)$ & \\
\hline \multicolumn{5}{|l|}{ Domínio 2} \\
\hline Baixo impacto & $134(43,9)$ & $171(56,1)$ & $305(100,0)$ & \multirow[b]{2}{*}{0,953} \\
\hline Alto/médio impacto & $35(44,3)$ & $44(55,7)$ & $79(100,0)$ & \\
\hline \multicolumn{5}{|l|}{ Domínio 3} \\
\hline Baixo impacto & $109(41,0)$ & $157(59,0)$ & $266(100,0)$ & \multirow[b]{2}{*}{0,072} \\
\hline Alto/médio impacto & $60(50,8)$ & $58(49,2)$ & $118(100,0)$ & \\
\hline \multicolumn{5}{|l|}{ Domínio 4} \\
\hline Baixo impacto & $145(42,6)$ & $195(57,4)$ & $340(100,0)$ & \multirow{2}{*}{0,135} \\
\hline Alto/médio impacto & $24(54,5)$ & $20(45,5)$ & $44(100,0)$ & \\
\hline
\end{tabular}

A Tabela 3 apresenta os valores descritivos do questionário $C P Q_{8-10}$, observa-se que os valores-mínimos nos 4 domínios foram 0 e os valores máximos variaram de 16 a 30 , sendo o domínio 1 o que apresentou a maior média $(5,02 \pm 3,48)$.

Tabela 3. Valores descritivos do questionário CPQ8-10

\begin{tabular}{|lccc|}
\hline CPQ8-10 & $\begin{array}{c}\text { Valor } \\
\text { Mínimo }\end{array}$ & $\begin{array}{c}\text { Valor } \\
\text { Máximo }\end{array}$ & Média \pm DP \\
\hline Variável & 0 & 16 & $5,02 \pm 3,48$ \\
\hline Domínio 1 & o & 18 & $3,37 \pm 3,58$ \\
\hline Domínio 2 & 0 & 20 & $4,1 \pm 4,25$ \\
\hline Domínio 3 & 0 & 30 & $4,18 \pm 4,91$ \\
\hline Domínio 4 & o & 72 & $16,7 \pm 12,75$ \\
\hline Impacto na Qualidade de vida & & &
\end{tabular}

DISCUSSÃO

De toda a amostra de escolares estudados, houve uma prevalência maior do 
sexo feminino, semelhante aos resultados encontrados nos estudos de Cabral et al. ${ }^{12} \mathrm{e}$ Ebenriter $^{14}$. Já Paiva et al. ${ }^{15}$ tiveram uma maior presença de escolares do sexo masculino, divergindo deste estudo e dos demais já citados. Com relação a idade, o presente estudo teve uma maior participação de crianças com idade de 8 anos e 9 anos em relação as crianças com 10 anos, dados aproximados com o estudo de Cabral et al. ${ }^{12}$ e Ebenriter ${ }^{14}$.

A prevalência dos traumatismos dentários vem sendo estudada em vários países $^{16-18}$ e os resultados geralmente evidenciam taxas elevadas. $\mathrm{Na}$ dentição permanente, a prevalência varia de $8 \%$ a $58,6 \%$. Esses números mostram as grandes variações nas prevalências encontradas ${ }^{19}$. No presente estudo a prevalência dos traumatismos dentários foi de $44 \%$ semelhante a encontrada no estudo de Marinho et al. ${ }^{20}$. Entretanto, outros estudos encontraram números menores ${ }^{14,21}$. Essa variação de prevalências verificada na literatura pode ser justificada por características culturais e educacionais diferentes nos diferentes locais nos quais os estudos foram desenvolvidos.

Em relação ao gênero, os traumatismos dentários têm sido mais frequentes em indivíduos do sexo masculino, afirmativa essa que vem sendo comprovada nas pesquisas ${ }^{21-24}$, concordando com os achados desse estudo. A literatura mostra que isso ocorre porque os meninos estão mais ligados a atividades esportivas ou brincadeiras em locais abertos, sendo assim mais expostos a acidentes intencionais ou não ${ }^{15,22}$.

No presente estudo embora os meninos tenham sido mais acometidos por lesões traumáticas, não houve diferença estatisticamente significativa entre a presença de trauma e os gêneros, este resultado concorda com 0 estudo de Paiva et. al. ${ }^{15}$. Traebert et al. ${ }^{25}$ justificam a falta de diferença estatística quando falam que as meninas estão praticando mais esportes e até algumas atividades que anteriormente eram mais relacionadas com o sexo masculino, tonando-se assim tão susceptíveis aos traumas dentais quanto os meninos.

Com relação a prevalência de traumatismo por idade, houve um número maior de crianças com 9 e 10 anos de idade acometidas por algum tipo de traumatismo, apresentando assim significância estatística $(p=0,042)$. Segundo Adreasen e Andreasen ${ }^{26}$ essa maior ocorrência dos traumas dentários em crianças entre 9 e 10 anos acontece porque nessa faixa etária as brincadeiras e atividades esportivas tornam-se frequentes. Já Carvalho et al. ${ }^{27}$ afirma que a primeira década de vida é a faixa etária mais acometida pelos traumatismos dentários, pois os traumas aumentam substancialmente com os primeiros esforços da criança para se mover, devido à falta de experiência e coordenação motora. Estes autores afirmam ainda que existem dois picos de incidência de trauma dentário, sendo um pico dos 2 aos 4 anos, seguido por um segundo pico dos 8 aos 10 anos de idade.

A literatura é unânime ao apontar os incisivos centrais superiores como sendo os dentes mais acometidos por traumatismos dentários, somente variando em relação aos valores percentuais apontados por diferentes autores $^{23}$. Em relação a esta análise, verificouse uma prevalência bastante elevada no grupo dos incisivos. Estes dados estão de acordo com os achados de outros estudos na literatura ${ }^{1,20,28}$.

Esta ocorrência pode ser devido ao fato destes dentes erupcionarem mais cedo que os incisivos laterais superiores, estando, portanto, sujeitos a um maior período de exposição a fatores de risco ${ }^{16}$. Além desses fatores, a localização dos incisivos centrais superiores na arcada dentária, também favorece o acometimento por traumas dentais ${ }^{23}$.

De acordo com Reis et al. ${ }^{29}$, a extensão e complexidade das lesões dentárias traumáticas dependem da natureza, da intensidade do trauma e características do dente envolvido. No presente estudo, o tipo de trauma mais frequente foi a fratura apenas de esmalte, estando esses dados em concordância com a literatura pesquisada ${ }^{17,21,28}$. O trauma apenas em esmalte é provavelmente o mais encontrado, por ser o de menor severidade nas classificações, não necessitando de um impacto tão severo para que aconteça ${ }^{17}$, se mostrando ser o tipo de lesão mais comum na dentição permanente ${ }^{30}$.

A luxação lateral foi o segundo tipo de trauma mais prevalente encontrado neste estudo, este resultado, por ter sido obtido em análise de dentição permanente, difere dos encontrados até então na literatura; uma vez que os estudos mostram as luxações sendo mais frequentes na dentição decídua, devido as características resilientes e porosas dos ossos maxilares nesta fase ${ }^{4}$. Já Paiva et al. $^{15}$ concordam com os achados deste estudo, quando afirmam que as fraturas coronárias e as luxações, são as lesões dentárias de maior prevalência, podendo acometer simultaneamente a polpa, os tecidos mineralizados e de sustentação do dente.

Sendo assim, a fratura apenas em 
esmalte não causa sérios danos ao elemento dentário e não necessita de tratamento imediato, já as luxações comprometem o tecido pulpar e os tecidos periodontais de proteção e suporte do dente, sendo desta forma, uma consequência grave do traumatismo dentário, necessitando de tratamento imediato e controle do caso.

É valido ressaltar que neste estudo houve associação estatisticamente significativa entre os traumatismos dentários e o impacto na qualidade de vida das crianças $(p=0,011)$. Na pesquisa desenvolvida por Cabral et al. ${ }^{12}$, os autores encontraram maior associação estatística entre os aspectos relacionados ao bem-estar social e necessidade de tratamento. $O$ estudo de Antunes et al. $^{31}$ demonstrou também que crianças e adolescentes que sofreram traumatismo apresentaram mais experiências negativas e maiores impactos na limitação funcional e bem-estar emocional quando comparadas com as que não sofreram.

Divergindo do presente estudo, o trauma não teve impacto na qualidade de vida das crianças na faixa etária de 8 a 10 anos de idade no estudo de Enberiter et al. ${ }^{14}$ e nem no estudo de Piovesan et al. ${ }^{32}$. Isto pode ser explicado pelo fato de o trauma mais frequente nos dois estudos ser a fratura de esmalte, que é menos severa do que os outros tipos de trauma encontrados ${ }^{14}$.

Alguns autores defendem que traumas leves não causam impactos negativos na qualidade de vida de crianças $^{33,34}$. Já Bendo et al. ${ }^{35}$ não só discordam de tal afirmativa como também relatam que, em casos de fraturas mais complexas, envolvendo dentina e comprometimento pulpar, aumentam em 2,4 vezes as chances do impacto negativo na qualidade de vida de crianças e adolescentes.

Alguns autores concordam com as respostas do $\mathrm{CPQ}_{8-10}$ deste estudo quando afirmam que crianças que sofreram traumatismo dentário tendem a relatar impacto na qualidade de vida, como dificuldade em se alimentar e fazer a higiene bucal, e acabam demonstrando mais vergonha de sorrir, sentem-se chateadas e isso interfere na fonação ${ }^{8,36}$. Destaca-se que a utilização exclusiva de métodos quantitativos tradicionais de avaliação bucal é considerada limitada, por não considerar os aspectos psicossociais e funcionais da saúde bucal ${ }^{37}$. Tornando-se essencial a análise da qualidade de vida, para compreender o verdadeiro impacto das alterações e complementar a avaliação clínica $^{38}$, sobretudo no cotidiano das crianças, pelo risco de comprometer as atividades diárias e o desenvolvimento futuro ${ }^{39}$.
CONCLUSÃO

Conclui-se que houve uma prevalência do sexo feminino participante do estudo, porém as crianças do sexo masculino apresentaram mais acometimento por traumas e não houve associação estatística com a ocorrência de traumatismo dentário. A faixa etária predominante foi de crianças com 9 anos participantes do estudo, no entanto, as crianças com idade de 9 e 10 anos apresentaram mais traumatismos dentário. O tipo de trauma mais prevalente foi o trauma apenas em esmalte seguido pela luxação lateral, sendo o grupo dos incisivos mais acometido por trauma. Houve impacto dos traumatismos dentários na qualidade de vida das crianças com idade de 8 , 9 e 10 anos que frequentam escolas da rede municipal da cidade de Patos-PB, sendo confirmado pela associação estatística entre estas variáveis.

\section{REFERÊNCIAS}

1. Mota LQ, Targino AGR, Lima MGGC, Farias JFG, Silva ALA, Farias FFG. Estudo do Traumatismo Dentário em Escolares do Município de João Pessoa, PB, Brasil. Pesq Bras Odontoped Clin Integr 2012;11(2):217-22.

2. Bastos JV, Cortes MIS. Traumatismo dentário. Arq Odontol. 2011;47(suppl2):80-5.

3. Sousa AM. Prevalência e fatores associados ao traumatismo dentário em adolescentes escolares [dissertação]. Recife: Programa de Pós-Graduação em Odontologia; Universidade Federal de Pernambuco; 2016.

4. Zaleckiene V, Peciuliene V, Brukiene V, Drukteinis S. Traumatic dental injuries: etiology, prevalence and possible outcomes. Stomatologija. 2014;16(1):7-14.

5. Andreasen JO, Andreasen FM. Essentials of traumatic injuries to the teeth. 2. ed. Copenhaggen: Munksgaard; 1990.

6. Mussarelli KR. Avaliação da casuística de fraturas coronárias e corono-radiculares do serviço de atendimento aos traumatismos dentários da FOP-UNICAMP [dissertação]. Piracicaba: Faculdade de Odontologia de Piracicaba, Universidade Estadual de Campinas; 2015.

7. Costa ABM. Traumatismos alvéolo-dentário: Avaliação dos conhecimentos e atitudes de uma amostra de professores do ensino fundamental do município de São Paulo [dissertação]. São Paulo: Faculdade de Odontologia de São Paulo, Universidade de São Paulo; 2004.

8. Antunes LAA, Leão AT, Maia LC. Impacto do traumatismo dentário na qualidade de vida de crianças e adolescentes: revisão crítica e instrumentos de medida. Ciênc Saúde Coletiva 2012;17(12):3417-24. 
9. Kirkwood BR. Essentials of medical statistics. 2. ed. Oxford: Blackwell Science; 1996.

10. Jokovic A, Locker D, Tompson B, Guyatt, G. Questionnaire for measuring oral health-related quality of life in eight- to ten-year-old children. Pediatr Dent. 2004;26(6):512-18.

11. Barbosa TS, Vicentin MDS, Gavião MBD. Qualidade de vida e saúde bucal em crianças Parte I: Versão brasileira do Child Perceptions Questionnaire 8-10. Ciênc Saúde Coletiva. 2011;16(10):4077-85.

12. Cabral RS, Branco CMC, Granville-Garcia AF, Firmino RT, Tôrres BO, Ferreira JMS. Impacto da saúde bucal na qualidade de vida de escolares do município de pequeno porte da Paraíba. Odonto (São Bernardo do Campo). 2016;23(45-46):47-55.

13. Fernandes MLMF, Moura FMP, Gamaliel KS, Correa-Faria P. Cárie dentária e necessidade de tratamento ortodôntico: impacto na qualidade de vida de escolares. Pesq Bras Odontopediatria Clin Integr. 2013;13(1):37-43.

14. Ebenriter CST. Impacto do trauma dental na qualidade de vida de crianças de 8 a 10 anos que frequentam escolas municipais de Florianópolis [monografia]. Florianópolis: Universidade Federal de Santa Catarina; 2017.

15. Paiva PCP, Paiva HN, Jorge KO, Filho PMO. Estudo transversal em escolares de 12 anos de idade sobre a necessidade de tratamento, etiologia e ocorrência de traumatismo dentário em Montes Claros, Brasil. Arq Odontol. 2013;49(1):19-25.

16. Cetinbas T, Yildirim G, Sonmez $H$. The relationship between sports activities and permanent incisor crown fractures in a group of school children aged 7-9 and 11-13 in Ankara. Turkey Dent Traumatol. 2008;24:532-6.

17. Fakhruddin KS, Kawas SA. Prevalence and etiological factors related to dental injuries amongst 18-22-year-olds in United Arab Emirates. Dent Traumatol. 2010;26:388-92.

18. Kramer PF, Feldens CA. Traumatismos da dentição decídua: prevenção, diagnóstico e tratamento. São Paulo: Santos; 2013.

19. Rodrigues AS, Castilho T, Antunes LAA, Antunes LS. Perfil Epidemiológico dos Traumatismos Dentários em Crianças e Adolescentes no Brasil. UNOPAR Cient Ciênc Biol Saúde. 2015;17(4): 267-78.

20. Marinho ACMR, Manso MC, Colares V, Andrade DJC. Prevalência de traumatismo dentário e fatores associados em adolescentes no concelho do Porto. Rev Port Estomatol Med Dent Cir Maxilofac. 2013:54(3):143-49.

21. Vieira EM, Cangussu MCT, Vianna MIP, Cabral MBB, Roque RN, Anjos ES. Prevalência, gravidade e fatores associados ao traumatismo dentário em escolares de 12 e 15-19 anos de idade em Salvador, Bahia. Rev Saúde Col UEFS. 2017:7(1):51-7.
22. Basha S, Mohammad RN, Swamy HS, Sexena V. Association between Traumatic Dental Injury, Obesity, and Socioeconomic Status in 6and 13-Year-Old Schoolchildren. Soc Work Public Health. 2015;30(4):336-44.

23. Doria CN, Soares NCL. Traumatismos em dentes anteriores na infância: importância do conhecimento sobre prevalência e etiologia para a prevenção [monografia]. Aracaju: Universidade Tiradentes; 2016.

24. Shappo ME, Mohr E, Almeida LH. O impacto das fraturas dentárias classe IV na vida de crianças e adolescentes. RFO UPF. 2018;22(3):321-25.

25. Traebert J, Marcon KB, Lacerda JT. Prevalence of traumatic dental injuries and associated factors in schoolchildren of Palhoça, Santa Catarina State. Ciên Saúde Coletiva. 2010;15:1849-55.

26. Andreasen JO, Andreasen FM, Anderson L. Textbook and color atlas of traumatic injuries to the teeth. 5. ed. Hoboken: Wiley-Blackwell; 2018.

27. Carvalho RG, Soares SR, Silva EJNL, Mendonça TA, Fonseca OHS, Antunes HS et al. Estudo epidemiológico das fraturas coronárias em pacientes atendidos em um projeto de trauma dental em um período de 6 anos. Rev Bras Odontol. 2013;70(1):4-7.

28. Bilder L, Margvelashvili V, Sgan-Cohen $H$, Kalandadze $M$, Levin L, Ivanishvili $R$ et al. Traumatic dental injuries among 12- and 15year-old adolescents in Georgia: results of the pathfinder study. Dent Traumatol. 2015;32(3):169-73.

29. Reis A, Loguercio AD, Kraul A, Matson E. Reattachment of fracture teet: a review of literatue regarding techniques and materials. Oper Dent. 2004;29(2):226-33.

30. Wright G, Bell A, McGlashan G, Vincent C, Welbury RR. Dentoalveolar trauma in Glasgow: an audit of mechanism and injury. Dent Traumatol. 2007;23(4):226-31.

31. Antunes LS, Debossan PF, Bohrer LS, Abreu FV, Quintanilha LE, Antunes LA. Impact of traumatic dental injury on the quality-of-life of children and adolescents: a case-control study. Acta Odontol Scand. 2013;71(5):1123-28.

32. Piovesan C, Abella C, Ardenghi TM. Child oral health-related quality of life and socioeconomic factors associated with traumatic dental injuries in schoolchildren. Oral Health Prev Dent. 2011;9(4):405-11.

33. Abanto J, Paiva SM, Raggio DP, Celiberti $P$, Aldrigui JM, Bönecker M. The impact of dental caries and trauma in children on family quality of life. Community Dent Oral Epidemiol. 2012;40(4):323-31.

34. Viegas CM, Paiva SM, Carvalho AC, Scarpelli AC, Ferreira FM, Pordeus IA. Influence of traumatic dental injury on quality of life of 
Brazilian preschool children and their families. Dent Traumatol. 2014;30(5):338-47.

35. Bendo CB, Paiva SM, Varni JW, Vale MP. Oral health-related quality of life and traumatic dental injuries in Brazilian adolescents. Community Dent Oral Epidemiol. 2014;42(3):216-23.

36. Vieira-Andrade RG, Siqueira MB, Gomes GB, D'Avila S, Pordeus IA, Paiva SM et al. Impact of traumatic dental injury on the quality of life of young children: a case-control study. Int Dent J. 2015;65(5):261-68.

37. Vargas-Ferreira F, Piovesan C, Praetzel JR, Mendes FM, Allison PJ, Ardenghi TM Tooth Erosion with Low Severity Does Not Impact Child Oral Health-Related Quality of Life. Caries Res. 2010;44(6):531-39.

38. Antunes LA, Castilho T, Marinho M, Fraga RS, Antunes LS. Childhood bruxism: Related factors and impact on oral health-related quality of life. Spec Care Dentist. 2016;36(1):7-12.

39. Schuch HS, Costa Fdos S, Torriani DD, Demarco FF, Goettems ML. Oral health-related quality of life of schoolchildren: impact of clinical and psychosocial variables. Int J Paediatr Dent. 2015;25(5):358-65.

\section{CONFLITO DE INTERESSES}

Os autores declaram não haver conflitos de interesse

\section{AUTOR PARA CORRESPONDÊNCIA}

Jéssica Fernanda Delfino dos Santos

Rua Antônio Gonçalves, 215 - Bairro Jatobá 58707-400 Patos - PB, Brasil

E-mail: jessica.fernanda812@gmail.com 